Running title: Perceived importance of prospective memory in TBI

\title{
Perceived importance of prospective memory failures in adults with traumatic
}

\section{brain injury}

Jia Huang ${ }^{\mathrm{a}}$, Jennifer Fleming *b,c,d ${ }^{\text {, Nadine L. Pomery }}{ }^{\mathrm{b}}$, Raymond C.K. Chan ${ }^{\mathrm{a}}$, David H.K. Shum ${ }^{\mathrm{e}}$

a. Neuropsychology and Applied Cognitive Neuroscience Laboratory, Key

Laboratory of Mental Health, Institute of Psychology, Chinese Academy of Sciences, Beijing, China

b. The University of Queensland, School of Health and Rehabilitation Sciences,

Australia

c. Occupational Therapy Department, Princess Alexandra Hospital, Brisbane, Australia

d. Centre for Functioning and Health Research, Metro South Hospital and Health Services, Brisbane, Australia

e. Behavioral Basis of Health Program, Griffith Health Institute and School of Applied Psychology Griffith University, Brisbane, Australia

*Correspondence should be addressed to:

Assoc Prof. Jennifer Fleming, School of Health and Rehabilitation Sciences, The University of Queensland. Brisbane, Qld, 4072. Email: j.fleming@uq.edu.au;

Tel: 6173896 3084; Fax: 61733651622 


\section{Abstract}

Prospective memory (PM) is the ability to carry out an intended action in the future.

Failures in PM are often observed as more frequent in individuals with traumatic

brain injury (TBI) than controls. However, it remains unknown how individuals with

TBI and their significant others perceive the importance of these PM problems. In the current study, four groups (38 TBI, 34 TBI-other, 34 controls, 31 control-other) were recruited to report on the perceived importance of PM failures using Part B of the Comprehensive Assessments of Prospective Memory (CAPM). Individuals with TBI perceived PM failures as being more important than their significant others did. Controls’ ratings did not differ from their significant others. There were no statistically significant differences in rated importance for PM problems involving the basic activities of daily living (BADL) component and those involving the instrumental activities of daily living (IADL) . Implications of the results are discussed in terms of the motivation of people with TBI.

Keywords: traumatic brain injury, prospective memory, comprehensive assessment of prospective memory (CAPM) 
Perceived importance of prospective memory failures in adults with traumatic

\section{brain injury}

Prospective memory (PM) is the ability to remember to perform an action at a specified later time (Cockburn, 1996; Mioni, Stablum, McClintock, \& Cantagallo). PM has been found to be impaired in TBI patients, both in adult (Shum, Levin, \& Chan, 2011; Umeda, Kurosaki, Terasawa, Kato, \& Miyahara, 2011) and child and adolescent samples .(McCauley, Pedroza, et al., 2011; McCauley, Wilde, et al., 2011).Even patients with uncomplicated mild TBI are more likely to report ongoing memory and concentration problems in daily activities than controls (Ponsford, Cameron, Fitzgerald, Grant, \& Mikocka-Walus, 2011). Sustained problems in everyday memory have been associated with poor quality of life following TBI (Hu, Feng, Fan, Xiong, \& Huang, 2012; Kinsella et al., 1996; Moran et al., 2011).and have implications for patients’ daily living such as compromised safety, reduced independence and productivity, and increased reliance on carers.

PM problems can be assessed using behavioral measures such as psychometric tests and experimental tasks (Wang et al., 2011; Wilson et al., 2005), which target cognitive processing and evaluate memory function in standard ways. PM can also be assessed using self- and other-rated questionnaires which target PM function in everyday life (Hannon, Adams, Harrington, Fries-Dias, \& Gipson, 1995; Mateer, Sohlberg, \& Crinean, 1987). These questionnaires provide an ecologically relevant way of assessing PM problems in clinical populations because they approach assessment from the everyday perspective of individuals and their significant others. 
The Prospective Memory Questionnaire (PMQ) was the first self-report questionnaire developed to assess PM in patients with brain injury (Hannon, et al., 1995). It assesses the frequency of problems in five dimensions of PM: Long-Term Episodic Tasks, Short Term Episodic Tasks, Long-Term Habitual Tasks, Short-Term Habitual Tasks and Techniques to Assist Recall. The authors of the PMQ found that patients with TBI reported difficulty with short-term habitual tasks but not with more long-term remembering (Hannon, et al., 1995). A second self-report test of PM is the Prospective and Retrospective Memory Questionnaire (PRMQ) developed by Maylor, Smith, Sala and Logie (2002). (Pavawalla, Schmitter-Edgecombe, \& Smith, 2012) reported a statistically significant difference in the prospective component of the PRMQ between control and TBI groups.

A third test designed to measure self-reported PM across the adult life span is the Comprehensive Assessment of Prospective Memory (CAPM) (Waugh, 1999). It includes three sections, with Section A assessing the frequency of PM failure, Section B the perceived importance of these lapses, and Section C the reasons for success and failure on the PM tasks. The same items are used to assess frequency in Section A and perceived importance in Section B. Waugh (1999) performed a principal components analysis of the items in Section A in a sample of older adults and found two reliable components, one involving basic activities of daily living (termed BADL) and the other instrumental activities of daily living (termed IADL). BADL includes items referring to self-care tasks such as dressing, eating, personal grooming and hygiene. IADL includes items referring to household management activities such as 
managing finances, shopping and meal preparation,, which are subject to more common PM failures than the BADL items (Roche, Fleming, \& Shum, 2002; Waugh, 1999). The same latent factor structure of IADL and BADL components has been found in a non-Caucasian sample (Chan, Qing, Wu, \& Shum, 2010).

The internal consistency of the two subscales based on the IADL and BADL components was 0.92 (BADL) and 0.79 (IADL) in Waugh's sample.. These estimates were largely confirmed in a study by Chau, Lee, Fleming, Roche, and Shum (2007), which also showed good rest-retest reliability for the two subscales. As with the other two tests of PM described above, the CAPM has been shown to differentiate patients with TBI and controls (Roche et al., 2002) In that study Section A of the CAPM was the focus of interest. Self-assessments of TBI patients were compared with the same assessments of their significant others (relatives or close friends) and with the assessments of controls and their significant others. Roche et al, reported that TBI patients underestimated the frequency of their PM failures compared to the assessment by their significant others, while healthy controls reported a similar frequency of PM failure to their significant others

To date, no published work has investigated differences between TBI patients and controls on Section B of the CAPM directed to the perceived importance of PM failures. Understanding self-perceptions may, however, contribute to the successful engagement of individuals with TBI in rehabilitation. Recognising the potentially serious consequences and personal significance of their PM failures may increase their motivation to avoid such problems in the future and purposefully use memory 
strategies.

Given that in the study of Roche et al. (2002), TBI patients were found to lack self-awareness and to underestimate their PM failures, we predicted that they may underestimate as well the importance of PM failure. Because data had been gathered in that study on response to Section B of the CAPM, we were able to test the prediction. We hypothesized that individuals with TBI perceive their PM failures to be less important than their significant others, whereas control participants rate the importance of PM failures as similar to that of their significant others. Second, we hypothesised that there are differences between the BADL and IADL subscales. Given that PM problems on IADL items are more frequent than those on BADL items, we hypothesised that individuals with TBI perceive problems in the IADL items of greater importance. Third, we hypothesized that, just as TBI patients and their significant others differ in their assessments of rated importance, TBI patients and normal controls differ in the rated importance of PM failure, with TBI patients' ratings indicating lesser importance.

Method

\section{Participants}

The participants were those described in Roche et al (2002). In total, 136 participants took part in the study, consisting of four subgroups: a TBI group paired with a group of significant others, referred to as the "TBI-other" group, and a control group paired with a group of significant others, referred to as the "control-other" 
group. The TBI group consisted of 38 adults who had sustained a severe TBI, as indicated by an initial Glasgow Coma Scale of less than 8 and a period of coma, or post-traumatic amnesia for longer than 24 hours. Significant others were close relatives or friends who were familiar with the patients with TBI or healthy controls. Table 1 summarizes the demographic characteristics of the four groups.

Additional characteristics of the TBI group were: mean weeks post-injury 56.6 (median $=37$, range 11 to 169); mean initial Glasgow Coma Scale score 5.1 (median $=2.4$, range 3 to 11 ); mean days in post-traumatic amnesia 62.6 (median $=42.8$, range 9 to 185), mean days in acute care 41.4 (median $=27.9$, range 5 to 106); mean days in rehabilitation 59.9 (median 56.9, range 0 to 228).

Inclusion criteria for the TBI group were: sufficient English communication skills to complete the questionnaire; past inpatients or current outpatients at a major metropolitan public hospital in Brisbane, Australia; a relative or friend available to do the other-ratings for them; living in the community for at least four weeks after discharge from inpatient care to ensure they had some post-injury experience outside of the hospital environment.

The 34 control participants were drawn from the researchers' local community and social networks. Inclusion criteria for the control group were: not from health related services; no history of brain injury; closely matched with the TBI group on age, gender, and years of education.

Inclusion criteria for both the TBI-other group and control-other group included being familiar with the participants' functional status (i.e., a close relative or friend) 
and being nominated by the TBI patients and control participants. The TBI-other group consisted of 14 mothers, 7 wives, 5 fathers, 4 husbands, 1 sister and 2 unspecified relationships (choosing 'other' item, but not specified). The control-other group consisted of 12 mothers, 9 wives, 3 husbands, 2 father, and 5 missing data (failure to choose 'other' item). No significant difference was found in the proportion of significant other relationships between the TBI-other and control-other groups. All significant others were required to rate how well they knew the participant. In the TBI-other group, 27 were rated as knowing the participant extremely well, 5 as very well, 1 as reasonably well, while in the control-other group, 16 were rated as knowing the participant extremely well, 6 as very well, 2 as reasonably well and 7 had missing data.

Insert Table 1 about here

\section{Measures}

Data reported here were gathered using Section B of the CAPM. Section B can be scored for two subscales, BADL and IADL, as described above and these two subscales were used., Section B assesses how much of a problem a PM failure is or would be for the individual rather than how often the problem occurs. The specific instruction was '.. this time we would like you to indicate how much of a problem these memory lapses have been. If you have not experienced some of these lapses, simply indicate how much of a problem they would be, if they were to happen to you.' 
On each of the items the participant is required to rate how much of a problem it is when or if a particular PM lapse occurs. The items are rated on a five-point scale from 1 ("no problem at all”), 2 (“a slight problem), 3 (“a moderate problem”), 4 (“aserious problem”), to 5 (“a very serious problem”).

The significant other's version of the questionnaire contains the same items as the original questionnaire, but asks the significant other to indicate how much of a problem the PM lapses have been for their relative/friend. If their relative/friend has not experienced some of these lapses, it asks them to simply indicate how much of a problem they would be, if they were to occur.

\section{Procedure}

Ethical clearance was obtained from both the hospital and university ethics committees. Before giving written consent, all participants and their significant others were provided with information about the purpose of the study and their participation. Most of the TBI participants and their significant others completed the CAPM either in a quiet non-distracting environment at the hospital. Occasionally, when it was inconvenient to come to hospital, the interview was conducted by telephone. Significant others completed the questionnaire independently at either the same time as the participants (TBI and control) or at home and returned the questionnaires via reply paid mail.

Because TBI patients were shown in the previous report to report lower frequencies of PM lapses, the analysis of importance sought to take this into account. 
For the TBI group only items that they or their significant others judged to occur once a month or more were included. The importance ratings of these items were averaged for each patient and for their significant others, and this was done separately for the BADL and the IADL subscale items. This was done to ensure that the ratings being compared were for actual PM lapses rather than for hypothetical ones. In the case of the control group and their significant others, ratings for all items on the BADL and IADL subscales were included.

\section{Data analysis}

The data were analyzed using the Statistical Package for Social Sciences (SPSS) for Windows Version 21.0.Less than 5\% of the data values were missing and a pair-wise deletion strategy was adopted in the analysis. The rating data were treated as continuous and subjected to parametric statistical tests, after checking that assumptions had been met within accepted tolerances. Repeated measures analysis of variance (ANOVA) was used for comparison between TBI, control, and their respective significant others and for the comparison of scores on the BADL and IADL subscales. An independent $t$ test was used for comparing TBI and control groups. A conventional alpha level of .05 was initially adopted but a Bonferroni adjustment in terms of the number of tests made (7) provided a final alpha level of .007. ..

Results

For the TBI group, the mean importance ratings (and 95\%CIs) for the BADL set of 
items were 3.03 (2.77 to 3.29) and for the IADL set were 2.84 (2.66 to 3.02). For their significant others, the comparable statistics were 1.82 (1.45 to 1.84) for the BADL set and 1.72 (1.40 to 2.04) for the IADL set. A two-way ANOVA (with repeated measures on both factors) on importance ratings showed a statistically significant effect for the comparison of TBI patient with significant others, $F(1 / 34)=47.75, p$ $<.001$, but not for the BADL-IADL difference, $F(1 / 34)=3.49, p=.07$ or the interaction, $F(1 / 34)=.306, p=.58$.

For the control group, the mean importance ratings (and 95\%CIs) for the BADL set were 2.10 (1.88 to 2.32 ) and for the IADL set were 2.27 (1.96 to 2.59). For their significant others the comparable statistics were 1.96 (1.65 to 2.26) for the BADL set and 1.96 (1.76 to 2.17) for the IADL set. A two-way ANOVA (with repeated measures on both factors) on importance rating showed no statistically significant effects: for the control-significant other comparison, $F(1 / 29)=1.79, p=.19$, for the BADL-IADL comparison, $F(1 / 29)=3.56, p=.07$, and for the interaction, $F(1 / 29)=$ $1.89, p=.18$

Given that there was no BADL-IADL difference, importance ratings for the two item sets were combined and the average composite ratings compared between TBI patients and controls using an independent two-sided $t$ test with no assumption of equal variances. The mean importance rating for the TBI group was 2.94 (95\% CI = 2.82 to 3.04 ) and for the control group was 2.12 ( 1.84 to 2.38). The difference was $.82(95 \% \mathrm{CI}=.53$ to 1.11$)$ and the $t$ value for the difference was $5.71(d f=36), p$ $<.001$. In terms of the standard deviation of the control group, the difference 
represents an effect size (Cohen’s $d$ ) of 1.12.

In summary, importance ratings were higher for the TBI group when compared to the importance ratings of both their significant others and the control group. The BADL-IADL difference was not statistically significant nor was the interaction of this factor with the patient - significant other difference.

\section{Discussion}

The clear result from the data analyzed here is that the TBI group rated the importance of PM failure more highly than their significant others and the control participants. The difference was statistically significant and was substantial. In terms of the widely used convention of effect size measurement, the difference would be considered a large effect (Cohen, 1988). In absolute terms, it constituted almost one unit of the five-unit scale or some $16 \%$ of the total scale. The difference was for items that either the TBI patient or their significant other reported occurred at least monthly. That is, the ratings for TBI patients were not of hypothetical lapses but of those that occurred with some frequency and thus have the potential to impact on the person's everyday living.

The result was not as we expected. Based on the findings of the analysis of Section A of the CAPM that the TBI patients judged the frequency of the occurrence of PM failures to be lower than that of their significant others or of controls, we expected that this apparent lack of self-awareness would be associated with a lower rating of the importance of these failures. The result indicates that the TBI patients 
attached more not less importance to these failures. In the light of this finding it may be that the perceived lower frequency of failures is a form of symptom minimization, an attempt to reduce the negative impact of the symptom on everyday functioning by reducing the perception of its occurrence. This may not be deliberate but a cognitive distortion that helps to preserve self-esteem: 'this is a serious problem but fortunately it does not occur very often'. Another plausible explanation in addition to the cognitive distortion was the higher demand of retrospective memory for frequency rating than for importance rating. Previous studies showed that retrospective memory was affected in TBI and the retrospective memory contributed significantly both to the retrospective and prospective cognitive components in patients with TBI (Clune-Ryberg et al., 2011). These of course are speculative, but suggest that it would be of value in subsequent work to explore the frequency-importance nexus of PM failure more thoroughly.

The higher rated importance of PM lapses for TBI patients may be a source of motivation for rehabilitation. The use of compensatory aids, such as memory books, depends on a willingness on the part of the patient to modify their behavior. We had previously expected that lack of self-awareness on the part of TBI patients of the frequency of PM lapses may militate against intervention efforts, but this may be offset by the importance attached to these lapses when they do occur. A practical implication is that score on Part B of the CAPM should predict uptake of compensatory methods.

We had hypothesized that there would be differences between the BADL and 
IADL subscales in terms of rated importance. In the analysis of Section A of the CAPM reported on earlier (Roche et al. 2002), there were differences between the subscales in terms of the frequency of occurrence of PM lapses, with the more complex and less habitual IADL items giving rise to estimates of more frequent occurrence. In the present study the IADL-BADL difference did not appear as a main effect or in interaction with participant status. There were differences between the means (e.g., higher rated importance for the IADL than the BADL subscale items for the TBI group) but the differences were relatively small and not statistically significant. We attempted an item analysis of the scales to identify particular items that might differentiate TBI from controls. However, the numbers of TBI patients varied from item to item, given our decision to score for each patient only items that were judged to be relatively frequent. This plus the relatively small $n$ s meant that estimates of differing reliability for each item were being compared. Larger samples than recruited for this study are required for meaningful item analysis. 


\section{Acknowledgements}

Preparation of this manuscript was partly supported by an Endeavor Research

Fellowship awarded to Jia Huang and an Australian Academy of Science Scientific

Visit to China Award to David Shum. 


\section{References}

Chan, R., Qing, Y., Wu, Q., \& Shum, H. (2010). Prospective memory in healthy Chinese people: The latent structure of the Comprehensive Assessment of Prospective Memory Questionnaire. Neuropsychological Rehabilitation, 20(3), 459-470.

Clune-Ryberg, M., Blanco-Campal, A., Carton, S., Pender, N., O'Brien, D., Phillips, J., . . Burke, T. (2011). The contribution of retrospective memory, attention and executive functions to the prospective and retrospective components of prospective memory following TBI. Brain Injury, 25(9), 819-831. doi: 10.3109/02699052.2011.589790

Cockburn, J. (1996). Failure of prospective memory after acquired brain damage: Preliminary investigation and suggestions for future directions. Journal of Clinical and Experimental Neuropsychology, 18(2), 304-309. doi: 10.1080/01688639608408284

Cohen, J. (1988). Statistical power analysis for the behavioral sciences(2nd ed.): Hillsdale, NJ: Lawrence Earlbaum Associates.

Hannon, R., Adams, P., Harrington, S., Fries-Dias, C., \& Gipson, M. T. (1995). Effects of brain injury and age on prospective memory self-rating and performance. Rehabilitation Psychology, 40(4), 289.

Hu, X. B., Feng, Z., Fan, Y. C., Xiong, Z. Y., \& Huang, Q. W. (2012). Health-related quality-of-life after traumatic brain injury: A 2-year follow-up study in Wuhan, China. Brain Injury, 26(2), 183-187. doi: 10.3109/02699052.2011.648707

Kinsella, G., Murtagh, D., Landry, A., Homfray, K., Hammond, M., O'Beirne, L., . . Ponsford, J. (1996). Everyday memory following traumatic brain injury. Brain Injury, 10(7), 499-508. doi: doi:10.1080/026990596124214

Mateer, C. A., Sohlberg, M. K. M., \& Crinean, J. (1987). Focus on clinical research: Perceptions of memory function in individuals with closed-head injury. The Journal of Head Trauma Rehabilitation, 2(3), 74-84.

Maylor, E. A., Smith, G., Sala, S. D., \& Logie, R. H. (2002). Prospective and retrospective memory in normal aging and dementia: An experimental study. Memory and Cognition, 30(6), 871-884.

McCauley, S. R., Pedroza, C., Chapman, S. B., Cook, L. G., Vasquez, A. C., \& Levin, H. S. (2011). Monetary incentive effects on event-based prospective memory three months after traumatic brain injury in children. Journal of Clinical and Experimental Neuropsychology, 33(6), 639-646. doi: 10.1080/13803395.2010.547844

McCauley, S. R., Wilde, E. A., Bigler, E. D., Chu, Z., Yallampalli, R., Oni, M. B., . . Levin, H. S. (2011). Diffusion tensor imaging of incentive effects in prospective memory after pediatric traumatic brain injury. Journal of Neurotrauma, 28(4), 503-516. doi: 10.1089/neu.2010.1555

Mioni, G., Stablum, F., McClintock, S. M., \& Cantagallo, A. Time-based prospective memory in severe traumatic brain injury patients: The involvement of executive functions and time perception. Journal of the International Neuropsychological Society, Available on CJO 2012, doi: 10.1017/s1355617712000306. doi: 10.1017/s1355617712000306

Moran, L. M., Taylor, H. G., Rusin, J., Bangert, B., Dietrich, A., Nuss, K. E., . . Yeates, K. O. (2011). Quality of Life in Pediatric Mild Traumatic Brain Injury and its Relationship to Postconcussive Symptoms. Journal of Pediatric Psychology, doi: 10.1093/jpepsy/jsr087. doi: 10.1093/jpepsy/jsr087

Pavawalla, S. P., Schmitter-Edgecombe, M., \& Smith, R. E. (2012). Prospective memory after 
moderate-to-severe traumatic brain injury: A multinomial modeling approach.

Neuropsychology, 26(1), 91-101. doi: 10.1037/a0025866

Ponsford, J., Cameron, P., Fitzgerald, M., Grant, M., \& Mikocka-Walus, A. (2011). Long-term outcomes after uncomplicated mild traumatic brain injury: a comparison with trauma controls. Journal of Neurotrauma, 28(6), 937-946. doi: 10.1089/neu.2010.1516

Roche, N. L., Fleming, J. M., \& Shum, D. H. K. (2002). Self-awareness of prospective memory failure in adults with traumatic brain injury. Brain Injury, 16(11), 931-945.

Shum, D., Levin, H., \& Chan, R. C. (2011). Prospective memory in patients with closed head injury: a review. Neuropsychologia, 49(8), 2156-2165. doi: 10.1016/j.neuropsychologia.2011.02.006

Umeda, S., Kurosaki, Y., Terasawa, Y., Kato, M., \& Miyahara, Y. (2011). Deficits in prospective memory following damage to the prefrontal cortex. Neuropsychologia, 49(8), 2178-2184. doi: 10.1016/j.neuropsychologia.2011.03.036

Wang, Y., Chan, R. C., Cui, J. F., Yang, T. X., Deng, Y. Y., Gong, Q. Y., \& Shum, D. (2011). Stability of prospective memory deficits in individuals with schizotypal personality traits. Psychiatry Research, 189(1), 156-157. doi: 10.1016/j.psychres.2011.01.002

Waugh, N. (1999). Self-report of the yound, middle-aged, young-old and old-old individuals oon prospective memory functioning. Honours Thesis, Griffith University, Brisbane, Australia.

Wilson, B. A., Emslie, H., Foley, J., Shiel, A., Watson, P., Hawkins, K., ... Evans, J. J. (Eds.). (2005). The Cambridge Prospective Memory Test(CAMPROMPT) Manual: Oxford, UK: Harcourt Assessment. 
Table 1

Descriptive statistics of demographic information among the four groups of participants

\begin{tabular}{|c|c|c|c|c|}
\hline & $\begin{array}{l}\text { TBI } \\
(n=38)\end{array}$ & $\begin{array}{l}\text { TBI-other } \\
(n=33)\end{array}$ & $\begin{array}{l}\text { Control } \\
(n=34)\end{array}$ & $\begin{array}{l}\text { Control-other } \\
(n=31)\end{array}$ \\
\hline Age (years) & 27.97 (10.53) & 44.31 (9.07) & $28.11(11.03)$ & $41.83(9.67)$ \\
\hline Education (years) & $10.89(1.90)$ & 11.83 (1.95) & $10.93(1.27)$ & 11.83 (2.77) \\
\hline Gender (Male\%) & $73.7 \%$ & $30.3 \%$ & $78.6 \%$ & $20 \%$ \\
\hline \multicolumn{5}{|l|}{ Marital status } \\
\hline Married\% & $36.8 \%$ & $81.3 \%$ & $48.1 \%$ & $100 \%$ \\
\hline Divorced\% & $2.6 \%$ & $12.5 \%$ & $0 \%$ & $0 \%$ \\
\hline Single\% & $57.9 \%$ & $3.1 \%$ & $48.1 \%$ & $0 \%$ \\
\hline
\end{tabular}


